\title{
Spectroscopy at HERA
}

\author{
Christiane Risler, on behalf of the ZEUS and H1 collaborations \\ DESY, Notkestrasse 85, D-22603 Hamburg, Germany
}

\begin{abstract}
Searches for strange and charmed pentaquark candidates by the ZEUS and H1 experiments in $e p$ collisions at HERA are presented. The analyses are based on deep-inelastic scattering and also photoproduction data at a center-of-mass energy of $300-318 \mathrm{GeV}$ using the full HERA I luminosity.
\end{abstract}

Keywords: deep inelastic scattering, spectroscopy, strange pentaquark, charm pentaquark

PACS: $14.20 .-\mathrm{c}, 14.20 . J n, 14.20 . \mathrm{Lq}, 14.80 .-\mathrm{j}$

\section{INTRODUCTION}

Recently observations of a narrow baryonic state decaying to $K^{+} n$ or $K_{S}^{0} p$ by fixed-target experiments at low center-of-mass energies have triggered intense experimental activity, followed by many observations but also non-observations by several experiments [1]. Results by the ZEUS and H1 experiments on searches for a strange pentaquark $\Theta^{+}$and also for a possible charm pentaquark in deep inelastic ep scattering (DIS) at HERA are presented here.

\section{STRANGE PENTAQUARK SEARCHES AT HERA}

A search for the strange pentaquark decaying to $K_{S}^{0} p(\bar{p})$ has been performed by the ZEUS experiment [2]. The analysed HERA-I data correspond to an integrated luminosity of $121 \mathrm{pb}^{-1}$. The $K_{S}^{0}$ mesons are identified through its decay into charged pions, and proton candidates are selected based on the measurement of the ionisation energy loss $\mathrm{d} E / \mathrm{d} x$ at proton momenta $p(p r)<1.5 \mathrm{GeV}$. The invariant mass distribution of $K_{S}^{0} p(\bar{p})$ combinations for photon virtualities $Q^{2}>20 \mathrm{GeV}^{2}$ is shown in Fig.1a. A fit of the sum of a background function and two Gaussian distributions yields a signal of $221 \pm 48$ events at a mass of $1521.5 \pm 1.5 \mathrm{MeV}$. The significance of the signal is estimated to be $4.6 \sigma$. The $\Theta^{+}$production cross section is extracted [3] in the kinematic region $Q^{2}>20 \mathrm{GeV}^{2}, 0.04<y<0.95, p_{T}\left(K_{S}^{0} p(\bar{p})\right)>0.5 \mathrm{GeV}$ and $\left|\eta\left(K_{S}^{0} p(\bar{p})\right)\right|<1.5$ and is found to be $\sigma_{v i s}=125 \pm 27$ (stat.) ${ }_{-28}^{+36}$ (syst.)pb. A similar analysis has been performed by the $\mathrm{H} 1$ experiment [4], using $71 \mathrm{pb}^{-1}$ of DIS data in the kinematic range defined by $0.1<y<0.6,5<Q^{2}<100 \mathrm{GeV}^{2}$. Protons are selected using particle identificiation with likelihood cuts based on the $\mathrm{d} E / \mathrm{d} x$ measurement. No signal is observed and $Q^{2}$ and mass dependent upper limits on the $\Theta^{+}$production cross section at $95 \%$ C.L. are extracted, which are found to vary between 30 and $90 \mathrm{pb}$. In order to compare the upper limits more directly to the observation by the ZEUS experiment, the analysis was repeated restricted to the kinematic range $p(p r)<1.5 \mathrm{GeV}$ and $Q^{2}>20 \mathrm{GeV}$. The 
a)

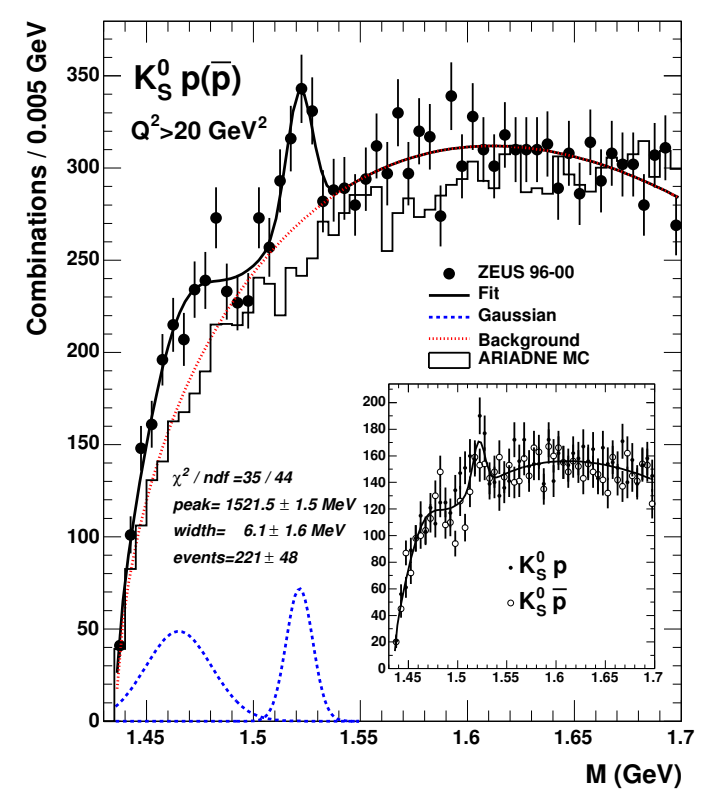

b)

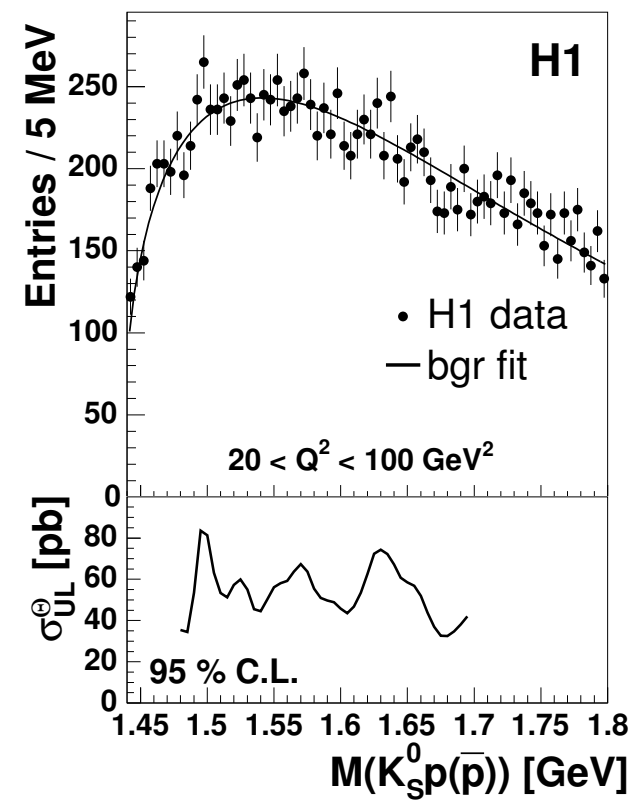

FIGURE 1. a) The invariant $K_{S}^{0} p(\bar{p})$ mass spectrum observed by ZEUS for $Q^{2}>20 \mathrm{GeV}^{2}$ together with a fit of a background function and two Gaussian distributions. b) The invariant mass spectrum and upper limit observed by $\mathrm{H} 1$ for $Q^{2}>20 \mathrm{GeV}^{2}$ and $p(p r)<1.5 \mathrm{GeV}$.

resulting invariant $K_{s}^{0} p(\bar{p})$ mass spectrum and upper limit are shown in Fig.1b. At a mass of $1522 \mathrm{MeV}$ an upper limit of $72 \mathrm{pb}$ is found, which does not exclude the cross section observed by ZEUS. ZEUS has also carried out a search for a resonance in the invariant $\Xi \pi$ spectrum, but no signal is found [5].

\section{CHARM PENTAQUARK SEARCHES AT HERA}

H1 has performed a search for a possible charmed pentaquark candidate and reported evidence for a narrow resonance in the $D^{*} p$ mass spectrum around $3.1 \mathrm{GeV}$ [6], shown in Fig.2a, using $75 \mathrm{pb}^{-1}$ of DIS HERA-I data at $1<Q^{2}<100 \mathrm{GeV}^{2}$. The $D^{*}-$ mesons are identified by evaluating the mass difference between the $D^{*}$ and the $D^{0}$, $\Delta M=M\left(K \pi \pi_{s}\right)-M(K \pi)$, and protons are selected via $\mathrm{d} E / \mathrm{d} x$ likelihoods. A fit to the data of a background function and a Gaussian yields $50.6 \pm 11.2$ signal events. The probability that the background distribution fluctuates to produce the signal is estimated from a fit using only the background function to be $4 \times 10^{-8}$, assuming Poisson statistics. The acceptance corrected ratio of the $D^{*} p$ to the $D^{*}$ cross section in the visible kinematic range is found to be $R_{c o r}\left(D^{*} p(3100) / D^{*}\right)=1.59 \pm 0.33_{-0.45}^{+0.33}$ [7]. ZEUS has performed a similar analysis using $126 \mathrm{pb}^{-1}$ of DIS and photoproduction $(\gamma p)$ data. The $D^{*}$ decay channels $K \pi \pi_{s}$ and also $K \pi \pi \pi \pi_{s}$ are investigated using a different kinematic range than that used by $\mathrm{H} 1$. No resonance is observed in the invariant $M\left(D^{*} p\right)$ spectra. The upper limit on the ratio of $D^{*}$-mesons orginating from $D^{*} p(3100)$ decays is extrated to be 0.59 $\%$ at $95 \%$ C.L. for the DIS sample at $Q^{2}>1 \mathrm{GeV}^{2}$ using the $K \pi \pi_{s}$ decay channel. The 
a)

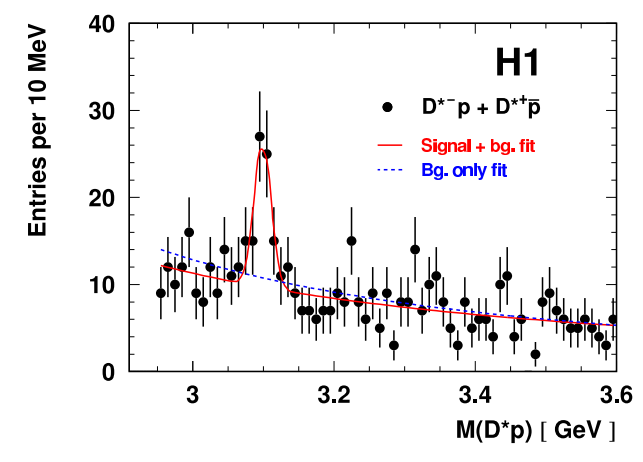

b)

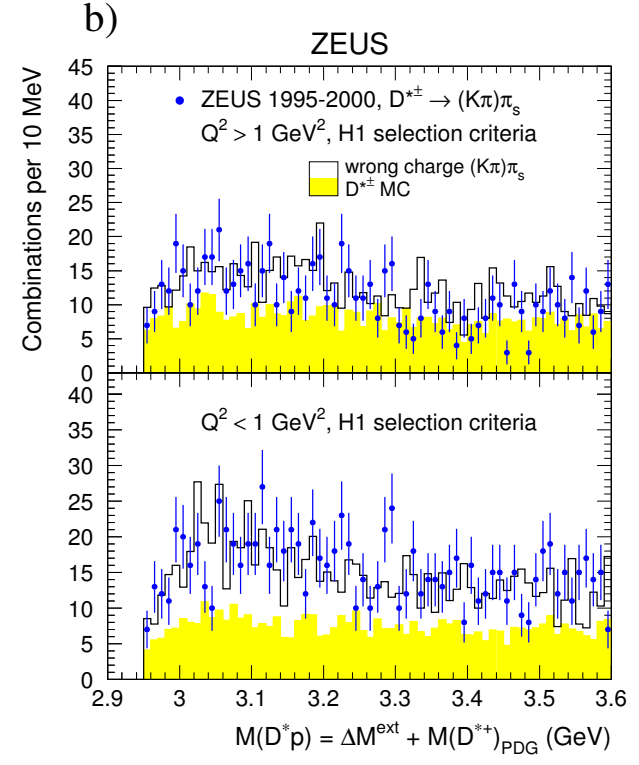

FIGURE 2. a) $M\left(D^{*} p\right)$ mass distribution from opposite-charge $D^{*} p$ combinations together with a fit of a background (dashed) and a background plus signal function(full line) measured by H1. b) $M\left(D^{*} p\right)$ mass distribution observed by ZEUS for DIS (top) and $\gamma p$ (bottom) events in the $K \pi \pi_{s}$ decay channel.

analysis has been repeated with selection cuts similar to those used by $\mathrm{H} 1$. The resulting mass spectra are shown in Fig.2b for the DIS (top) and the photoproduction sample (bottom). No signal is observed and the $\mathrm{H} 1$ observation is not confirmed by ZEUS.

\section{CONCLUSIONS}

The observation of a $\Theta^{+}$candidate by ZEUS can not be confirmed by $\mathrm{H} 1$, but the extracted upper limits on the production cross section do not exclude this observation. While $\mathrm{H} 1$ observes a narrow baryonic resonance decaying to $D^{*} p$, ZEUS does not. The ongoing high-luminosity data taking at HERA-II, may help to clarify these open questions.

\section{REFERENCES}

1. K. Hicks, Prog Part. Nucl. Phys. 55 (2005) 647 [hep-ex/0504027].

2. S. Chekanov et al. [ZEUS Collaboration], Phys. Lett. B 591, 7 (2004) [hep-ex/0403051].

3. S. Chekanov et al. [ZEUS Collaboration], contributed paper no. 10-0273 to 32nd International Conference on High Energy Physics (ICHEP04), August 2004, Beijing, China.

4. A. Aktas et al., [H1 Collaboration], accepted by Phys. Lett. B, [hep-ex/0604056].

5. S. Chekanov et al. [ZEUS Collaboration], Phys. Lett. B 610, 212 (2005) [hep-ex/0501069].

6. A. Aktas et al. [H1 Collaboration], Phys. Lett. B 588, 17 (2004) [hep-ex/0403017].

7. A. Aktas et al. [H1 Collaboration], contributet paper 401 to XXII International Symposium on LeptonPhoton Interactions at High Energy, June 2005, Uppsala, Sweden.

8. S. Chekanov et al. [ZEUS Collaboration], Eur. Phys. J. C 38, 29 (2004) [hep-ex/0409033]. 